\title{
HOLOMORPHIC FUNCTIONS ON A BANACH SPACE
}

\author{
BY SEÁN DINEEN ${ }^{1}$
}

Communicated by Raoul Bott, February 2, 1970

1. Unless otherwise stated we shall use the definitions and notation of [4]. $E$ will always be a complex Banach space and $\mathfrak{H C}(E)$ will denote the set of all complex valued holomorphic functions on all of $E . \theta$ will be a holomorphy type $[4$, p. 34$]$ and $\left(\mathcal{F}_{\theta}(E), J_{\omega, \theta}\right)$ will denote the topological vector space of holomorphic functions associated with $\theta$ as described in $[4, \mathrm{pp} .35,43] . c_{0}^{+}$will denote the set of all sequences of positive real numbers which tend to zero at infinity. $\mathfrak{K}$ will denote the set of all convex balanced compact subsets of $E$. Let $B_{1}$ be the closed unit ball of some fixed norm which defines $E$. \|\|$_{U}$ will denote the Minkowski functional of the subset $U$ of $E$.

An $\alpha$-holomorphy type $\theta$ is a holomorphy type whose definition depends only on the topological vector space structure of $E$ (i.e., it is independent of the actual norm used to define $E$ ) and if $U, V \subset E$ and $C$ is a positive real number such that $C U \subset V$ then for each $n$ we have

$$
C^{n}\left\|P_{n}\right\|_{\theta, U} \leqq\left\|P_{n}\right\|_{\theta, V} \quad \text { for all } P_{n} \in \mathcal{P}_{\theta}\left({ }^{n} E\right)
$$

where $\left(\rho_{\theta}\left({ }^{n} E\right),\|\|_{\theta}\right)$ is the $(n+1)$ st member of the sequence given by $\theta$ (see $[4$, p. 34]).

DEFinition 1. (a) Let $\theta$ be an $\alpha$-holomorphy type then $H_{\theta}(E)$ is the set of all elements of $\mathfrak{H C}(E)$ for which

(1) $\hat{d}^{n} f(0) \in P_{\theta}\left({ }^{n} E\right)$.

(2) For each $K \in \mathfrak{K}, \exists \epsilon>0$ such that

$$
\sum_{m=0}^{\infty}\left\|\frac{\hat{d}^{m} f(0)}{m !}\right\|_{\theta, K+\epsilon B_{1}}<\infty .
$$

(b) A seminorm $p$ on $H_{\theta}(E)$ is $\theta$-ported by $K \in \Re$ if, for each $\epsilon>0$, $\exists C(\epsilon)>0$ such that

AMS Subject Classifications. Primary 4630, 4645; Secondary 3202.

Key Words and Phrases. Holomorphy type, nuclear type, current type, Borel transform, formal power series.

1 The results in this paper are taken from the author's doctoral thesis written under the supervision of Professor Leopoldo Nachbin and presented at the University of Maryland, June 1969. It was written while the author was on leave of absence at Instituto de Matemática Pura e Aplicada, Rio de Janeiro, Brazil. The author acknowledges financial assistance from the Conselho Nacional de Pesquisas of Brazil and the National University of Ireland. 


$$
p(f) \leqq C(\epsilon) \sum_{m=0}^{\infty}\left\|\frac{\hat{d}^{m} f(0)}{m !}\right\|_{\theta, K+\epsilon B_{1}} \quad \text { for all } f \in H_{\theta}(E) .
$$

(c) The topology $T_{\theta}$ on $H_{\theta}(E)$ is that generated by all seminorms $\theta$ ported by some element of $\pi$.

For the nuclear type this is the topology considered in [6].

THEOREM 1. (a) Let $f=\sum_{n=0}^{\infty}\left(\hat{d}^{n} f(0) / n !\right) \in \mathcal{H C}(E)$ and $\hat{d}^{n} f(0)$ $\in \odot_{\theta}\left({ }^{n} E\right)$ for $n=0,1, \cdots$. Then the following conditions are equivalent:

(1) $f \in H_{\theta}(E)$.

(2) For each $K \in \mathcal{K},\left(\alpha_{n}\right)_{n=0}^{\infty} \in c_{0}^{+}$, we have

$$
\sum_{n=0}^{\infty}\left\|\frac{\hat{a}^{n} f(0)}{n !}\right\|_{\theta, K+\alpha_{n} B_{1}}<\infty .
$$

(3) For each $K \in \mathfrak{K},\left(\alpha_{n}\right)_{n=0}^{\infty} \in c_{0}^{+}$, we have

$$
\lim _{n \rightarrow \infty}\left\|\frac{d^{n} f(0)}{n !}\right\|_{\theta, K+\alpha_{n} B_{1}}^{1 / n} .
$$

(b) The $T_{\theta}$ topology on $H_{\theta}(E)$ is generated by all seminorms of the form

$$
p(f)=\sum_{n=0}^{\infty}\left\|\frac{d^{n} f(0)}{n !}\right\|_{\theta, K+\alpha_{n} B_{1}}
$$

as $K$ ranges over $\mathfrak{K}$ and $\left(\alpha_{n}\right)_{n=0}^{\infty}$ ranges over $c_{0}^{+}$.

(c) $\left(H_{\theta}(E), T_{\theta}\right)$ is a complete topological vector space.

Taking $\theta$ as the current type we get a global definition for elements of $\mathfrak{H C}(E)$.

THEOREM 2. Let $\left(P_{n}\right)_{n=0}^{\infty}$ be a sequence of continuous polynomials, $P_{n}$ being homogeneous of degree $n$, then $\sum_{n=0}^{\infty} P_{n}$ is the Taylor series expansion of an element of $\mathcal{H C}(E)$ if and only if for each $K \in \mathcal{K}$ and $\left(\alpha_{n}\right)_{n=0}^{\infty}$ $\in c_{0}^{+}$we have

$$
\lim _{n \rightarrow \infty}\left\|P_{n}\right\|_{K+\alpha_{n} B_{1}}^{1 / n}=0 \text {. }
$$

Remarks. (1) We do not know if $\left(H_{\theta}(E), T_{\theta}\right)$ is a bornological space. However we can get the following result:

The bornological topology on $H_{\theta}(E)$ associated with $T_{\theta}$ is the finest locally convex topology on $H_{\theta}(E)$ for which the Taylor series converges absolutely and which induces on each space $P_{\theta}\left({ }^{n} E\right)$ its original norm topology. 
(2) For any $\alpha$-holomorphy type $\left(H_{\theta}(E), T_{\theta}\right) \subset\left(\mathcal{F C}_{\theta}(E), J_{\omega, \theta}\right)$ continuously. For the current type $\theta$ we get $H_{\theta}(E)=\mathfrak{H}_{\theta}(E)=\mathscr{H C}(E)$ and $T_{\theta}=J_{\omega, \theta}$.

2. We denote by $N$ the nuclear type which in a certain sense (see [7]) can be looked upon as a maximal type.

Definition 2. An $\alpha$-holomorphy type $\theta$ is an $\alpha$ - $\beta$-holomorphy type if

(1) For each $n, \odot_{\theta}\left({ }^{n} E\right) \supset \odot_{N}\left({ }^{n} E\right)$ and $\left\|P_{n}\right\|_{\theta, U} \leqq\left\|P_{n}\right\|_{N, U}$ for all $P_{n} \in \odot_{N}\left({ }^{n} E\right)$, and all $U \subset E$.

(2) For each $\left.n, \mathcal{P}_{f}{ }^{(} E\right)($ see $[5])$ is a dense subspace of $\left(\mathcal{P}_{\theta}\left({ }^{n} E\right),\|\|_{\theta}\right)$.

For $\alpha$ - $\beta$-holomorphy types we get a Banach space isomorphism between $\left(\mathscr{P}_{\theta}\left({ }^{n} E\right),\|\|_{\theta}\right)^{\prime}$ and a subspace of $\odot^{\left({ }^{n} E^{\prime}\right)}$ which we denote by $\left(\mathcal{P}_{\theta^{\prime}}\left({ }^{n} E^{\prime}\right),\|\| \theta^{\prime}\right)$.

The Borel transform of $T \in\left(H_{\theta}(E), T_{\theta}\right)^{\prime}, \hat{T}$, is a function defined on $E^{\prime}$ by $\hat{T}(\phi)=T(\exp \phi)$. The Borel transform gives a one-to-one correspondence between elements of $\left(H_{\theta}(E), T_{\theta}\right)^{\prime}$ and a vector space of holomorphic functions on $E^{\prime}$ which we shall call the $\theta^{\prime}$-compact exponential type in $E$.

To consider partial differential operators we put further conditions on $\alpha$ - $\beta$-holomorphy types and get what we call $\alpha-\beta-\gamma$-holomorphy types. The conditions are for the most part natural and one is a slightly weaker form of the requirement that $\left(\Theta_{\theta^{\prime}}\left({ }^{n} E^{\prime}\right),\|\| \theta^{\prime}\right)_{n=0}^{\infty}$ be a holomorphy type. We then define the Fréchet space of $\theta$-formal power series, $\Im P_{\theta}(E)$.

THEOREM 3. If $\theta$ is an $\alpha-\beta-\gamma$-holomorphy type and $Q$ is a partial differential operator on $\mathfrak{F} \mathcal{P}_{\theta}(E)$ then

(1) All solutions of $Q$ (i.e., $\forall f$ such that $Q(f)=0$ ) can be approximated in $\mathcal{F P}_{\theta}(E)$ by $\theta$-exponential polynomial solutions.

(2) If $Q \neq 0$ then $Q$ maps $\mathfrak{F} \mathcal{P}_{\theta}(E)$ onto itself.

We will publish in a future paper full proofs of the above theorems and assertions together with some related results and examples.

\section{REFERENCES}

1. C. P. Gupta, Malgrange's theorem for nuclearly entire functions of bounded type on a Banach space, Notas de Matemática, no. 37, Inst. Mate. Pura Apl., Rio de Janeiro, 1966.

2. J. Horváth, Topological vector spaces and distributions. Vol. 1, Addison-Wesley, Reading, Mass., 1966. MR 34 \#4863.

3. A. Martineau, Sur les fonctionnelles analytiques et la transformation de FourierBorel, J. Analyse Math. 11 (1963), 1-164. MR 28 \#2437.

4. L. Nachbin, Topology on spaces of holomorphic mappings, Springer-Verlag, Berlin and New York, 1968. 
5. - Convolution operators in spaces of nuclearly entire functions on a Banach space, Proc. Conference Functional Analysis and Related Topics in Honor of M. H. Stone, Springer-Verlag, Berlin and New York, (to appear).

6. L. Nachbin and C. Gupta, On Malgrange's theorem for nuclearly entire functions (to appear).

7. F. Treves, Topological vector spaces, distributions and kernels, Academic Press, New York, 1967. MR 37 \#726.

The Johns Hopkins University, Baltimore, Maryland 21218 